\title{
Atlas der Schweiz - Ein Rückblick
}

Am 20. Februar 1978 wurde im Naturhistorischen Museum in Bern die Fertigstellung des "Atlas der Schweiz" gefeiert. Die Herren Bundesrat Dr. Hans Hürlimann, Prof.Dr.h.c. Eduard Imhof (Chefredaktor), dipl. Ing. Ernst Huber (Direktor der Eidgenössischen Landestopographie) und Prof.Dr. Claude Raffestin (Präsident der Geographischen Gesellschaft der Schweiz) drückten in kurzen Ansprachen ihre Genugtuung über das abgeschlossene Werk aus. Mit der anschliessenden Eröffnung einer Ausstellung in der nahen Eidgenössischen Landesbibliothek wurde der fertige Atlas der Deffentlichkeit vorgestellt. Mit Recht waren alle Beteiligten zufrieden, und auch die vielen Besprechungen in den Massenmedien lauteten durchwegs anerkennend, ja begeistert. Erfreut war man auch zu vernehmen, dass nach diesem ersten Abschluss eine Weiterführung des Atlasses gesichert ist.

Es mag für unsere jüngeren Berufskollegen von Interesse sein, einiges über die reichlich bewegte Vorgeschichte des Atlasses zu vernehmen, von den Erwartungen zu hören, die wir an den Atlas knüpften, von den Erfahrungen, die wir machten und die vielleicht für künftige Gemeinschaftsarbeiten nützlich sein könnten. Dabei wird früher Gesagtes teils etwas anders gewichtet $(7,8$, 9), teils im Interesse der Abrundung dieses Berichtes wiederholt (5).

Der Atlas ensprang der Initiative von Delegierten der "Geographischen Gesellschaft der Schweiz", damals 'Verband Schweizerischer Geographischer Gesellschaften", im folgenden kurz "Verband" genannt. In den Delgiertenversammlungen war das Thema Atlas immer wieder aufgegriffen, aber nur wenig der Realisierung entgegengeführt worden. Einen bedeutsamen Fortschritt brachten 1953 die unter dem damaligen Verbandspräsidenten Hans Annaheim beschlossenen neuen Verbandsstatuten. Sie sahen neben den üblichen Vereinsorganen eine Forschungskommission vor. Aeusserer Anlass für die Schaffung dieser Kommission war ein Brief des Präsidenten der Schweizerischen Naturforschenden Gesellschaft vom Sommer 1953 gewesen. Darnach setzte die Möglichkeit, Bundesbeiträge für erdkundliche Forschungsarbeiten auszulösen voraus, dass eine Forschungskommission ein nationales Forschungsprogramm aufstellte, die Arbeiten koordinierte und für deren wissenschaftlich einwandfreie Durchführung die Verantwortung übernahm. Dabei war in erster Linie an Gemeinschaftsarbeiten gedacht. Mit der Schaffung des Schweizerischen Nationalfonds (1952) ist eine derartige Forschungskommission ohnehin unerlässlich geworden. Die von den Verbandsdelegierten bereits 1952, also schon bevor die neuen Statuten in Kraft gesetzt waren, eingesetzte Forschungskommission bestand zunächst aus nur fünf Mitgliedern (Annaheim, Biermann, Gutersohn als Vorsitzender, Perret, Vosseler), wurde dann aber während der Erweiterung der Projekteliste vergrössert und in Subkommsissionen gegliedert. Eine dieser Subkommissionen war die bereits bestehende "Gruppe Landesatlas". Weil man nach damaligen Erwartungen vor allem mehrere Gemeinschaftsarbeiten verwirklichen wollte, die Atlasarbeiten dagegen sich immer wieder verzögerten, entstand schliesslich eine - zweifellos zu lange - Liste möglicher Projekte. Leider war ihnen kein sichtbarer Erfolg beschieden. Doch soll hier an sie erinnert werden, denn in diesem weiteren Rahmen gesehen ist unsere Genugtuung über das glückliche Gelingen des Atlasprojektes umso verständlicher.

\section{Gemeinschaftsarbeiten}

= $=$ = = = = = = = = = = = = =

Die Beteiligten waren sich klar darüber, dass die geplanten Arbeiten neben den wissenschaftlichen Arbeiten der einzelnen Geographen geleistet werden mussten und daher für jeden ein $\mathrm{Ab}-$ wägen zwischen individueller und kollektiver Tätigkeit voraussetzten. Mit Gemeinschaftsarbeiten, die womöglich eine weitere Oeffentlichkeit ansprechen sollten, wollte man auch - und dies schien uns in jenen Jahren notwendiger als je namentlich die Stellung der Geographie im Bereich der übrigen Wissenschaften stärken. An den Mittelschulen stand das Fach Geographie im Rahmen des Eidgenössischen Maturitätsreglementes ohnehin ständig unter Druck, indem von verschiedener Seite eine Reduktion seiner Wochenstundenzahl und die Vorverlegung oder gar Abschaffung der Maturitätsprüfung postuliert wurde, eine Drohung, der nur mit fachlichen Leistungen sowohl in der Schule als auch in der Deffentlichkeit begegnet werden konnte.

Die folgenden Projekte wurden in den Sitzungen der Forschungskommission vorgelegt und diskutiert: Exkursionsführer, Gebietsbeschreibungen, Fichier, Geographisches Lexikon, Geographie der Schweiz.

Prof. Dr. Heinrich Gutersohn, Schanzackerstr. 29, 8006 Zürich 


\section{Exkursionsführer}

Einen Exkursionsführer zur Hand zu haben, etwa entsprechend dem Geologischen Führer der Schweiz (Basel 1932), war ein häufig gehörter Wunsch namentlich der Geographielehrer. Einen formellen Vorschlag legte 1938 Haas (6) dem Verein Schweizerischer Geographielehrer vor; in der anschlies senden regen Diskussion wurde beschlossen, einige Beispiele ausarbeiten zu lassen. Wirth (21) publizierte 1939 einen entsprechenden Vorschlag. Im Schosse der Forschungskommission griff Annaheim, in der Folge stets ein besonders engagierter Förderer (1), den Gedanken auf und konkretisierte ihn im Einvernehmen mit Winkler. Die beiden legten 1955 ein Werkprogramm vor. Nach zähen Diskussionen wurden im Konzept voneinander abweichende Beispiele unterbreitet: Grosjean über das Schwarzenburgerland (3), Annaheim und Bartsch über das Gempenplateau (2), schliesslich Meylan über das Jouxtal (13). Weitere, leider nicht gedruckte Vorschläge reichten Nigg (Die bündnerische Herrschaft) und Merian (Das Engelbergertal) ein. Im Sinne praktischer Erprobung wurden die Exkursionen Schwarzenburgerland und Gempenplateau unter Leitung der genannten Autoren durchgeführt; unmittelbar anschliessend waren Diskussionen geplant, die aber aus Zeitmangel entweder überhaupt nicht oder nur verkürzt stattfanden. Die Resonanz war teils zustimmend, teils mit starken Vorbehalten verknüpft, auf alle Fälle für die Initianten wenig ermutigend. Nicht geklärt blieben namentlich die Fragen: Routenoder Regionalführer; bestimmt für die Hand des Lehrers, des Schülers mittlerer Reife, des gebildeten Nichtgeographen; Publikation als Ganzes oder in einzelnen, beispielsweise den Geographica Helvetica beigefügten Faszikeln; Finanzierung. Die Bemühungen erlahmten, und in einer Sitzung anfangs 1965 musste beschlossen werden, die Vorbereitungen vorläufig zu sistieren.

\section{Gebietsbeschreibungen}

Im Zusammenhang mit seiner eingehenden Beschäftigung mit den wissenschaftlichen Problemem der Landesplanung und angeregt durch die in der Bundesrepublik Deutschland bereitgeste1lten Grundlagen in Form von Kreisbeschreibungen und Planungsatlanten regte Winkler schon 1953 Regionalbeschreibungen an (19). Mit Recht wies er darauf hin, dass Aemter, Verbände, Forscher und neuerdings namentlich Landesplaner inmer wieder den Mangel landeskundlicher Dokumentation zu beklagen hatten. Dieser Mangel gab der Geographie eine Chance, sich durch Anregung, Planung und Schaffung derartiger Dokumentationen über Gemeinden, Regionen, Kantone usw. einzuschalten und damit der Oeffentlichkeit einen begehrten Dienst zu leisten. Die Forschungskommission begrüsste diese Initiative umsomehr, als die auf detaillierten Feldaufnahmen fussenden Publikationen auch für den Landesatlas und für weitergehende natur- und kulturgeographische Studien von hohem Wert gewesen wären. Fragen der Abgrenzung gegenüber den Projekten Fichier und Geographisches Lexikon sowie gegenüber den Datenbankarbeiten des Instituts für Orts-, Regional- und Landesplanung an der Eidgenössischen Technischen Hochschule Zürich brachten die Bemühungen ins Stocken.

\section{Fichier}

Aufgrund ähnlicher Ueberlegungen und Zielsetzungen wie sie Winkler für die Gebietsbeschreibungen vorgelegt hatte, schlug Liniger, damals Vertreter der Société Neuchâteloise de Géographie, 1954 mit viel Begeisterung den "Fichier" vor. Umfassende Angaben über Gemeinden, Regionen, Kantone usw. sollten redigiert und als Einzelblätter oder -hefte in rascher Folge publiziert werden. Der Initiant gedachte, der Forschungskommission Muster vorzulegen, liess indessen die vorgesehenen Fristen verstreichen. Später (1965) ersuchte er, ohne die Kommission einzuschalten, den Schweizerischen Nationalfonds um eine finanzielle Starthilfe, die jedoch abgelehnt wurde. Als weitere zugesagte Beispiele nicht eintrafen, konnte die Forschungskommission, schon im Hinblick auf die andern Projekte, den Fichier nicht weiter verfolgen.

\section{Geographisches Lexikon}

Und wiederum ähnliche Zielsetzungen wie für Gebietsbeschreibungen und Fichier galten für ein neues Geographisches Lexikon der Schweiz. Wir wussten, dass das von 1902 bis 1910 in Lieferungen erschienene und zu 6 Bänden vereinigte Lexikon seinerzeit einem Bedürfnis entsprochen hatte und deshalb ein verlegerischer Erfolg war, von dem auch die Geographie mindestens ideell profitierte. Wäre eine Neuherausgabe möglich, natürlich unter der Voraussetzung, dass ein neues Lexikon strukturell anders hätte konzipiert sein müssen? Die damals zum Beispiel für die Gemeinden dominierenden statistischen Angaben hätten durch fundierte natur- und kulturgeographische Fakten ergänzt werden müssen. Im Auftrag der Forschungskommission sprachen wir (Gutersohn, Paillard, Perret) 1963 mit dem Besitzer des Verlages Attinger in Neuenburg. Wir erfuhren, dass Attinger seinen damals erheblichen Gewinn mit dem in der Folge 1921 - 1934 in ähnlicher Aufmachung herausgegebenen Historisch-Biographischen Lexikon der Schweiz - es hatte in gewissen Einzelheiten den geographischen Forderungen eher besser entsprochen als das Geographische Lexikon-wieder eingebüsst hatte. So war die Aufnahme unseres Anliegens eher lau, es hätten zum voraus beträchtliche Geldmittel flüssig gemacht werden müssen. Weitere Bemühungen unterblieben. 
Eher am Rande der Beratungen dachte man in der Kommission auch an eine Neubearbeitung der "Geographie der Schweiz" von Jakob Früh, dies namentlich eingedenk der Tatsache, dass Früh seinerzeit vom Verband "mit dem ehrenvollen Auftrag betraut wurde, eine neuzeitlichen Anforderungen genügende, einheitlich erarbeitete Länderkunde der Schweiz zu schaffen." Frühs Werk wurde seinerzeit vom Bund finanziell unterstützt. Wie Früh, allerdings namentlich durch seine Altersbeschwerden bedingt, andere Geographen als Mitarbeiter beigezogen hatte, so dachten wir auch zum vornherein an die Zusammenarbeit mehrerer Geographen. Die Aufgabe wurde jedoch von der Forschungskommission angesichts der im Vordergrund stehenden Projekte Landesatlas und Exkursionsführer nicht weiter verfolgt.

Weshalb der, natürlich immer vom Atlas abgesehen, bedauerliche Misserfolg all dieser Pläne? Der Gründe sind viele: Man hätte sich zweifellos mit der nötigen Zielstrebigkeit auf eine Aufgabe, beispielsweise auf den in den Vorarbeiten weit fortgeschrittenen Exkursionsführer beschränken müssen, neue Aufgaben erst nach dem Abschluss der ersten in Angriff nehmen dürfen. Aber die Vielheit der Projekte ergab sich aus den zahlreichen fachlichen Diskussionen, die ja stets mit entsprechenden Anregungen und fast immer auch mit Offerten einzelner Kollegen zur Bereitstellung von Mustern beziehungsweise Beispielen verbunden waren. Man war sich der Zersplitterung der Kräfte bewusst, wollte aber keine wachen Initiativen vorzeitig abtöten. Als Hindernis erwiesen sich die häufigen Absenzen bei wichtigen Sitzungen; Geographen sind oft während Wochen und Monaten auf Studienreisen und nicht gewillt, die Verarbeitung der anfallenden Forschungsergebnisse zugunsten der Gruppenarbeit hintan zu stellen. Auch haben alle ihr volles Mass an Pflichten am eigenen Arbeitsplatz zu erfüllen. Vieles wäre leichter gewesen, wenn man Mitarbeiter hätte einspannen können, aber hiefür, wie auch für die anfallenden administrativen Kosten, feh1te einfach das Geld. Nicht verschwiegen sei, dass es fachliche Gegensätze und gelegentlich Spannungen gab, die anfängliche Begeisterung wich bei manchem einer bedauerlichen Resignation. Schliesslich ist die alte Erfahrung hervorzuheben: Es können noch so gute Pläne für kollektive Arbeit aufgestellt werden, letzten Endes kommt es stets darauf an, dass ein Einzelner die Sache an die Hand nimmt und unter Berücksichtigung der gefallenen Voten und der vereinbarten Richtlinien die eigentliche Arbeit leistet. Andererseits ist im Rückblick mit Genugtuung festzuhalten, dass das häufige Zusammensein fachlich anregend war, und dass hier manche persönliche Freundschaft erblühte, die den Beteiligten hohen Gewinn brachte.

Und nun zum Atlas:

\section{$==$ =ニニニニニ=ニ=ニ=ニ==}

\section{Vorgeschichte}

Ueber Vorläufer des Atlas der Schweiz berichteten Roth (14) und Imhof (11). Vosseler war wohl der erste, der in seinen Berichten über den Atlas de France im Jahre 1934 auf die Wünschbarkeit eines modernen Schweizerischen Landesatlasses hinwies (15). Vorschläge, teils mit Inhaltsverzeichnissen und Maquetten, wurden hierauf in den Fachsitzungen der "Sektion Geographie und Kartographie der Schweizerischen Naturforschenden Gesel1schaft" sowie an verschiedenen Delegiertenversammlungen des Verbandes vorgelegt. Sie verdichteten sich spürbar im Zusammenhang mit der Schweizerischen Landesausstellung 1939 und konkretisierten sich in mehreren Abhandlungen von Winkler $(17,18)$, Vosseler $(16)$, Imhof (7), Gutersohn (4). Auch der Verlag Kümmerly \& Frey legte einen Vorschlag mit Maquette vor.

Ziel des Atlasses ist es, der Wissenschaft, den Behörden, den Schulen und einer weiteren Oeffentlichkeit, also allen informationsbedürftigen Zeitgenossen und nicht zuletzt den Freunden guter Karten eine breit angelegte geographische Orientierung über die Schweiz zu vermitteln. Zu den Benützern sollten insbesondere auch die Landesplaner gehören, bieten sich ihnen doch die vielen thematischen Karten als äusserst reichhaltiges Grundlagenmaterial an. Alle diese Benützer sollen beim Studium der Karten sensibilisiert werden für die vielfältigen bekannten und weniger bekannten Wandlungen, denen unsere Schweizer Landschaften dauernd unterworfen sind.

Im Jahre 1941 wurde Eduard Imhof vom Verband offiziell beauftragt, einen Inhaltsplan mit Kostenvoranschlag auszuarbeiten. Dass Imhof bei der Schaffung des Atlasses eine massgebliche Rolle zu spielen haben werde, war den Geographen klar. Für ihn als Wissenschafter und Kartenautor war die thematische Karte seit Jahren ein faszinierendes Problem gewesen. Zahlreich und eng waren stets seine Kontakte mit den Schweizer Geographen, so auch bei den didaktischen Ueberlegungen zur Redaktion des Schweizer Mittelschulatlasses und der zahlreichen Schulhand- und -wandkarten. In allen diesen Fällen wusste sich Imhof den Forderungen der Fachvertreter loyal anzupassen. Bei der Erstellung seiner Karten hatte er sich im Zuge der Generalisierung und der Reliefzeichnung immer wieder mit orographisch-morphologischen, mit siedlungsstrukturellen und mit allgemein kulturgeographischen Tatsachen und Erscheinungen auseinanderzusetzen, stets auch im Hinblick auf die Forderung, ein bestimmtes Objekt optimal darzustellen. Diese engen Beziehungen mit dem Objekt der Geographie und mit den Geographen selber haben sich erfreulicherweise in den Jahren der Verwirklichung des Landesatlasses noch vertieft, zweifellos zum Nutzen beider Partner.

Vom Zeitpunkt jenes ersten Auftrages an sollten nicht weniger als zwanzig Jahre verstreichen, 
bis schliesslich der Start erfolgen und weitere siebzehn Jahre bis der glückliche Abschluss des Werkes gefeiert werden konnte! Der Ursachen, welche die Arbeiten hemmten, waren viele: $\mathrm{Zu}-$ nächst war die Mehrzahl der Beteiligten durch viele Aktivdienste blockiert, Imhof selber ausserdem dưrch andere Aufträge belastet. Im Schosse der Forschungskommission wurden immer wieder neue Aenderungswünsche laut. Eine drastische Verzögerung aber brachte die sich aufdrängende Forderung, die Aufnahmen und die ersten Blätter der Neuen Landeskarte, mit welcher neue Reproduktionsmethoden gekoppelt waren, abzuwarten. So lag Imhofs vorläufig endgültiger Organisationsund Inhaltsplan mit Maquette für 74 Tafeln erst 1958 vor. Hiezu wurden wiederum schriftliche Meinungsäusserungen der Schweizer Geographen erbeten. Parallel liefen Kontakte mit Verlägen, Erfolg versprechende namentlich mit dem EcycliosVerlag, einer Verlagsgemeinschaft, die 1945 1948 das "Schweizer Lexikon" herausgegeben hatte. Auch andere Fühler wurden ausgestreckt. Es waren vor allem die hohen budgetierten Kosten von rund 2,5 Mio Franken, welche einen Vertragsabschluss erschwerten. Wohl hätten den Ausgaben Einnahmen aus dem Verkauf gegenüber gestanden, überdies durften mit guten Gründen Beiträge des Bundes, des Nationalfonds sowie weiterer Fonds erwartet werden, aber alle diese Budgetposten fussten auf unsicheren Grundlagen. So verliefen manche Verhandlungen immer wieder im Sande, und die Einsatzfreudigkeit der Beteiligten wurde arg strapaziert.

Und doch gelang schliesslich der entscheidende Durchbruch! Fühlungsnahme von Imhof und Gutersohn (damals zugleich Präsident des Verbandes und der Forschungskommission), beide an der ETHZ, mit Prof. Dr. Hans Pallmann, Präsident des Schweizerischen Schulrates, sowie mit dipl.Ing. Ernst Huber, Direktor der Eidgenössischen Landestopographie, und Dr. Anton Meli, Direktor des Eidgenössischen Statistischen Amtes, eröffneten neue Perspektiven. Pallmann ebnete den Weg für eine Audienz bei Herrn Bundesrat Dr. Hansneter Tschudi, Vorsteher des Eidgenössischen Departementes des Innern, dem wir vier am 5. Dezember 1960 unser Anliegen unterbreiten konnten. Tschudi nahm den Vorschlag sehr positiv auf und ermunterte uns zu einer offiziellen, fundierten Eingabe an den Bundesrat. Diese Eingabe ging $a b$, wurde vom Schweizerischen Schulrat befürwortet und hatte Erfolg; am 25. Juli 1961 erklärte der Bundesrat den Atlas zu einer Aufgabe des Bundes und beschloss zugleich die Herstellung, Finanzierung und Herausgabe. Eine unter der Leitung von Chefredaktor Imhof stehende Redaktionskommission wurde ernannt, für die Bearbeitung das Kartographische und das Geographische Institut der ETH Zürich und für den Druck und die Herausgabe die Eidgenössische Landestopographie in Wabern ermächtigt. Ein der ETH angegliedertes Atlasbüro besorgte die vorbereitenden kartographischen Arbeiten. Das Schwergewicht war nun also, ohne dass man diesen Weg anfänglich gesucht hatte, auf die ETH und die Landestoptographie übergegangen. Doch wurde im Schosse des Verbandes selbstverständlich laufend über die Arbeiten berichtet und weitere Anregungen entgegengenommen.

Mit Zirkularen und in persönlichen Kontakten wurden alle Schweizer Geographen erneut eingeladen, sich in irgend einer Form als Autoren oder Mitarbeiter zu betätigen. Die im allgemeinen spontane Bereitschaft zur Zusammenarbeit war erfreulich. Freilich gab es auch Kollegen, die sich aus irgendwelchen Gründen nicht beteiligen wollten, aber im ganzen darf doch gesagt werden, dass der Atlas ein Dokument der Zusammenarbeit der Schweizer Geographen geworden ist. Für zahlreiche Tafeln aus Nachbargebieten der Geographie hatte die Redaktion von sich aus die einschlägigen Fachinstitutionen und aus diesen kompetente Fachleute zu suchen, auch dies mit sichtlichem Erfolg. Immer wieder traf man sich zu Besprechungen, namentlich der Chefredaktor hatte damit neben der eigentlichen technischen Arbeit viel Zeit zu investieren. Im übrigen sei hier auf die ersten Berichte Imhofs $(10,11)$ und auf das Atlasvorwort verwiesen.

\section{Kartentypen}

Der Atlas enthält thematische und eine kleine Auswahl topographischer Karten. Als Massstab der Hauptkarten resp. -tafeln, die das Format des Werkes indirekt bestimmten, erwies sich 1:500000 als zweckmässig. Einmal gefaltet ergeben die Tafeln eine handliche, leicht in der zugehörigen Sammelschachtel zu versorgende Kartensammlung. Auch für manche lineare Vergleiche mit Karten anderer Massstäbe ist das gewäh1te Mass praktisch. Ein erster Auftrag an die Landestopographie bestand nun darin, aufgrund der neuesten, eben abgeschlossenen Detailaufnahme unseres Landes eine neue Uebersichtskarte der Schweiz zu schaffen, die als Tafel 1 Bestandteil des Atlasses geworden ist, und die für den Grossteil der weiteren Tafeln als Grundlage diente. Unser Hauptmassstab ist im Vergleichmit den Basiskarten anderer Nationalatlanten verhältnismässig gross, was ja für ein kleines Land wie die Schweiz auch sein muss. Doch hat dies zur Folge, dass der Autor seine Karte detaillierter aufzubauen hat, dass er weniger generalisieren kann und also seine Forschung entsprechend mehr ins Einzelne vortreiben, dass er mehr Substanz erfassen muss. Dieser Umstand ruft nach einer entsprechenden Verpflichtung, der sich kein Autor entziehen kann, es sei denn, er redigiere relativ leere, stärker generalisierte, unnötig aufgeblasene, aber auch entsprechend oberflächlichere Karten.

Neben den grossen Tafeln enthält der Atlas viele Karten kleinerer Massstäbe. Solche Massstäbe, etwa 1:800000, wurden verwendet in allen jenen Fällen, in denen das Wissen für den grösseren Massstab einfach fehlte, der kleine Massstab also genügend Aussagekraft zu vermitteln vermochte; dies gilt für viele sogenannte Nebenkarten. Der Entscheid über die Massstabswahl lag beim Chefredaktor. Für ihn war die Wahl des kleineren Mass stabes dann ein heikler Entscheid, wenn der Autor mit Ueberzeugung eine Hauptkarte erwartet 
hatte, seine gelieferten Materialien aber hiefür nicht die nötige Dichte des Wissens brachten.

Gleichzeitig galt es zu entscheiden, welche Kartenunterlage, beispielsweise das Relief, das Gewässernetz, die politisch-administrative Region, und in welchem Flächenkolorit zu wählen sei.

Liegt das Ergebnis, die fertige Karte einmal vor so wird dem Betrachter des Bildes kaum mehr bewusst, wie sehr diese Entscheide und Auswahlkriterien für die Aussagekraft des fertigen Bildes massgebend waren.

Unser Atlas enthä1t wie jeder Nationalatlas sowohl analytische als auch synthetische Karten. So wie die erdkundliche Wissenschaft bei der Darstellung ihrer Studienobjekte, das heisst der Landschaft, in der Regel zunächst analysierend Landschaftselemente, nämlich Naturgrundlagen wie auch Elemente der Kulturlandschaft herauszuheben pflegt, so verfährt man auch bei der Konzipierung des Atlaswerkes. $\mathrm{Zu}$ den analytischen Karten gehören solche der reinen Forschung, etwa über Orographie, Geologie, Naturvegetation, um nur einige aus dem Bereich der Naturlandschaft zu nennen, daneben aber auch selektive Darstellungen der Bevölkerungsverteilung und -dichte, von Siedlungen, von Wirtschaftsformen als Ergebnisse von Leistungen, die unsere Gesellschaft in Gang halten, und viele andere. Dieses analytische Kartenprogranm wird ergänzt durch synthetische Tafeln, welche Zusammenhänge verschiedener Phänomene, Wechselwirkungen von vie1leicht zunächst nur zwei Elementen, gegenseitige Bedingtheiten aufzeigen und auf ihre Weise den Weg zum Verständnis von der Landschaft immanenten Zusammenhängen ebnen. Die umfassendere, in der Regel mehrere oder sogar viele Landschaftselemente einbeziehende Synthese, gleichsam eine Integration höherer Ordnung, bringt erst die eigentliche synthetische Karte. Je mehr Elemente in diesem reichhaltigen Spiel der Wirkungen und Gegenwirkungen mittun, die schliesslich das Wesen der Landschaft ausmachen, unso gewichtiger wird das Problem der Relativierung und der Wertung dieser verschiedenen Bausteine der Landschaften, eine Wertung, die für die Konzipierung der Karte nötig ist, aber stets dem subjektiven Ermessen des Bearbeiters anheim gestellt bleibt. Zweifellos stellen sich hier wesentlich höhere methodische Anforderungen sowohl an den Kartenautor als auch an den Betrachter des fertigen Werkes.

Eine für die Schweiz bekannte Tatsache bestätigte sich beim Redigieren der Karten erneut: die Dominanz des Reliefs im Beziehungsgefüge der Landschaft. Vielen Karten musste das Relief als Unterlage dienen, nämlich immer dort, wo sich eine gewichtige Relation zwischen Relief und andern Landschaftselementen manifestiert. Eng mit den Relief hängt natürlich das Gewässernetz zusammen, und so ist dieses dann auch vielen Karten untergelegt worden. Ein Versuch, die Synthese der Naturelemente voll zu erfassen, ergab auch dies nicht überraschend -, dass für die Klassierung der Naturlandschaften ebenfalls das
Relief dominiert (T.78). Eine Karte der Schwei-. zer Kulturlandschaften, also eine noch umfassendere synthetische Karte, stand lange auf dem Programm, konnte aber leider nicht realisiert werden; Ueberlegungen über die Konzeption einer derartigen Karte stecken doch wohl erst in den Anfängen. Ein entscheidender Fortschritt darf mit dem in Aussicht stehenden Planungsatlas (20) erwartet werden. Erfreulich ist, dass mit der Darstellung historischer Wandlungen ausgewählter Landschaften in Form der Gegenüberstellung älterer mit neuesten topographischen Karten Entwicklungen an ausgezeichneten Beispielen demonstriert werden konnten (T.22 u.a.).

Der Atlas enthält auch eine beschränkte Auswahl topographischer Karten, d.h. Blätter verschiedener Massstäbe der Landeskarte. In erster Linie ist die bereits erwähnte Haupttafe1 1:500000 (T.1) zu nennen, welche die Landestopographie rechtzeitig für den Atlas fertigstellte. Ihr Relief und ihr Gewässernetz, aber auch weitere ihrer Signaturen bilden die Unterlage der Haupttafeln.

Manche Karte gehört zu Fachbereichen, die nur 10se mit der Geographie zusammenhängen; doch wurden sie namentlich auch im Hinblick auf die angestrebte breite Verwendbarkeit des Werkes und im Streben nach interdisziplinärer Zusammenarbeit aufgenommen. Der Atlas soll ja nicht in erster Linie der Geographie, sondern auch den Nachbarwissenschaften und einer breiteren Oeffentlichkeit dienen. Einzuräunen ist freilich, dass mancher Atlasbenützer möglicherweise infolge dieser Zugaben das Wesen der Geographie verkennt und den oft vermuteten kompilatorischen Charakter unseres Faches scheinbar bestätigt findet. Manche Vertreter von Nachbarwissenschaften erhielten also die Möglichkeit, ihre neuesten Forschungsergebnisse im Rahmen unseres Atlasses zu publizieren. Dies gilt für eine Reihe von Tafeln der Abschnitte K1ima, Geophysik, Bodennutzung, Geschichte, Sprachwissenschaft u.a.. Für die Schweizerische Geologische Kommission ergab sich die erwünschte Gelegenheit, eine neue geologische Karte 1:500000 (T.4) sowie eine tektonische Karte (T.5) beizusteuern. Die Geomorphologen dürfen stolz sein auf ihre geomorphologischen Karten in Form einer grossen Landesübersicht (T.8) und ausgewählter Typenlandschaften (T.9). Auf sich allein gestellt hätten die genannten Wissenschafter ihre Karten aus rein finanziellen Gründen nicht so rasch herausbringen können. Eine erfreuliche Nebenwirkung dieser Mitarbeit von Nachbarwissenschaften waren dabei die mit der Programmierung und endgültigen Redaktion notwendigen persönlichen Kontakte zwischen ihnen und der Redaktion und damit auch mit der Geographie. Es darf füglich behauptet werden, dass diese $\mathrm{Zu}-$ sammenarbeit anhand eines gemeinsamen und hochgesteckten Zieles das Verständnis der Wissenschafter für die Anliegen der Kartographen und Geographen ganz wesentlich zu fördern vermochte, sicher zum Vorteil aller Beteiligten. 


\section{$\underline{\text { Programm }}$}

In bezug auf das Progranm sei auf das Inhaltsverzeichnis im Atlas hingewiesen. Es liegt auf der Hand, dass die frühesten, aus den dreissiger Jahren stammenden Vorschläge während der Vorbereitungsphase immer wieder neu auftretenden Kriterien angepasst, also abgeändert werden mussten. Und ebenso ging es unserem, der seinerzeitigen Eingabe an den Bundesrat zugrundegelegten Programm (11), freilich bei diesem nur noch in den Gliedern der grossen Tafelgruppen. Manchmal wurde von den ursprünglich vorgesehenen Kartenmassstäben abgewichen, wenn es sich bei der Bearbeitung der abgelieferten Grundlagen zeigte, dass das Objekt ebensogut, ja graphisch befriedigender in einem kleineren Massstab dargestellt werden konnte. Aber auch das Umgekehrte trat ein, wenn die Fü1le des zur Verfügung stehenden Materials einen grösseren Massstab, oder aber die Beschränkung auf einen kleineren Landschaftsausschnitt nahelegte. Und schliesslich - eine Tatsache, auf die Imhof bereits in seinem ersten Zirkular an die potentiellen Mitarbeiter hingewiesen hatte-: nicht alle geographischen Stoffe oder Ergebnisse eignen sich zur Darstellung in Kartenform; für gewisse Tatsachen ist die Tabelle oder die Graphik immer noch aussagekräftiger und wohl auch richtiger als die thematische Karte.

Programmänderungen mussten ausserdem vorgenommen werden, wenn es galt, einzelne Sachbereiche entsprechend ihrer Bedeutung im Gesamtrahmen entweder auszuweiten, noch öfter aber einzuschränken. Nicht wenige Mitarbeiter fanden im Zuge der Redigierung ihrer Entwürfe, es wären noch ergänzende Karten oder wenigstens einige Detailkarten zur Vertiefung der Darstellung ihres Anliegens notwendig. Dies ist durchaus verständlich. Für den Redaktor und seine Kommission war es in manchen Fällen nicht leicht, hier die ursprüngliche Konzeption starr beizubehalten, denn mehr Tafeln bedeuten auch Mehrumfang und Mehrkosten, und ausserdem wäre die Vertretung der verschiedenen Wissensbereiche nicht mehr genügend ausgewogen gewesen. Dagegen sind manche dieser Wünsche in einer Liste vermerkt, die bei der Weiterführung und Ergänzung des Atlasses herangezogen und neu überprüft werden soll. In diesem späteren Stadium wird es für die Geographen wichtig sein, mit neuen Vorschlägen bereit $\mathrm{zu}$ sein und sich für geographisch fundierte Ausgewogenheit auch des erweiterten Atlasprogramms einzusetzen.

\section{Koordinationsprobleme}

In Frage gestellt wurde das Programm des öftern ausserdem in den Fällen, wo mehrere Autoren ko1lektiv für eine einzelne Karte eingesetzt waren. Hier stellte sich dem Chefredaktor immer wieder die heikle Koordinationsaufgabe, denn dass sich dabei unterschiedliche Auffassungen offenbarten, ist verständlich. Nehmen wir als Beispiel Tafel 8 , Geomorphologie. Imhof hat über seine diesbezüglichen Erfahrungen berichtet (12). Die geominphologische Karte setzt sich aus Aufnahmen von Teilgebietên der Schweiz zusammen, die je einem Fachmann zugewiesen waren. Nun offenbarte sich begreiflicherweise an den Nahtstellen der einzelnen Mitarbeiterbereiche, dass in bezug auf die Interpretation gewisser Tatsachen, wie etwa die Qualifikation einer Moräne oder einer Terrasse Meinungsverschiedenheiten bestanden, die im persönlich-fachlichen Gespräch ausgeglichen werden mussten. Ueberdies ergaben sich beträchtliche Unterschiede in der Dichte der Aufnahmen und im Grad der vorgeschlagenen Generalisierung. Dies heisst nicht, dass der eine Forscher in seiner Feldarbeit weniger sorgfältig als sein Kollege gewesen war, sondern einfach, dass die Partner die Möglichkeiten der kartographischen Darstellung ihrer Studienobjekte unterschiedlich beurteilten. Ueberdies zeigte sich bei dieser $\mathrm{Ge}-$ legenheit, dass noch keine volle Uebereinkunft in bezug auf die anzuwendende Legendur erreicht war, obwohl seinerzeit die geomorphologische Arbeitsgruppe der Forschungskommission als ersten Punkt ihrer Studien die Schaffung zweckmässiger und verbindlicher Legenden angestrebt hatte. Gleiches gilt für gewisse Bereiche der Kulturgeographie; Vereinbarungen über zweckmässige Legenduren fehlen, auch ausländische Kollegen lieferten bisher lediglich Beispiele - ein Ansporn $z u$ entsprechender zielgerichteter Zusammenarbeit!

\section{Legenden und Kommentare}

Die Legenden der Tafeln sind, wie es für einen Nationalatlas der Schweiz notwendig und richtig ist, in drei, vereinzelt bei Einbezug des Rätoromanischen, in vier Sprachen (T.27, 28) abgefasst. Diese wenn auch knappen Texte benötigen Raum, und es war oft nicht leicht, ihn so auszusparen, dass das Kartenbild nicht übermässig belastet oder gar gestört wurde. Namentlich aber legten die Uebersetzungen von Fachausdrücken in vielen Fällen Probleme bloss, die man im aufgetretenen Mass nicht erwartet hatte. Dass mancher Begriff in der einen Sprache wohl klar, in einer andern aber unsicher, schillernd, vielleicht sogar umstritten ist, weiss man; und da die Autoren als Spezialisten ihres Faches nicht immer derselben Meinung wie die in ihrem Bereich sicher ebenfalls kompetenten Uebersetzer waren, ergaben sich grundsätzliche Auseinandersetzungen, die an sich wohl interessant waren, aber doch das Zeitprogramm und die Geduld des Chefredaktors strapazierten. Dasselbe gilt für die Kommentare.

Zweck der von den Autoren verfassten, zu jeder Tafel gehörenden Kommentare ist es, dem Kartenbetrachter darzulegen, welche spezielle Aussage angestrebt ist und welche Ueberlegungen zur gewählten Darstellung führten. Hinweise auf besonders markante Ergebnisse, die dem ungewohnten Atlasbenützer möglicherweise entgehen könnten, Hinweise auch auf ergänzende Tatsachen und auf verwandte Atlastafeln, ' ausserdem Tabellen und graphische Darstellungen sind weitere Bestandteile der Kommentare. Diese sehr frei gehandhabte Wegleitung war nicht immer leicht durchzusetzen. Gelegentlich wurde einfach eine auf knappstem Raum gefasste Abhandlung über den Kartengegen- 
stand verfasst, eine Art Extrakt aus einem Lehrbuch des in Frage stehenden Wissensbereiches, also die Gelegenheit nicht wahrgenommen, den speziellen Karteninhalt und die besondere Aussage dieser Karte herauszustellen, was natürlich auch in Anbetracht des knappen zur Verfügung stehenden Raumes ohnehin nicht leicht war. Ueberdies traten auch bei den Kommentaren die oben erwähnten Uebersetzungsschwierigkeiten auf, so dass die Beschaffung der Texte und hernach die administrativen Wege hin und zurück zu den einzelnen Autoren und Uebersetzern samt den notwendigen Rückfragen oder ergänzenden Besprechungen sehr viel Zeit beanspruchten.

\section{Erfolg und Ausblick}

Aufgrund der vielen Reaktionen auf das Erscheinen der einzelnen Atlaslieferungen darf man vorbehaltlos feststellen, dass dem Werk in den bei der seinerzeitigen Programmierung anvisierten, weitgestreuten Benützerkreisen ein erfreulicher Erfolg beschieden ist. Der Oeffentlichkeit wird mit dem Atlas die Vielheit der wissenschaftlichen Aspekte der Geographie dargelegt, den Geographen selber vermittelte er die Möglichkeit, Forschungsergebnisse in gediegener Form zu publizieren, die fruchtbare sachliche und personelle Zusammernarbeit mit den Kartographen und mit den Vertretern zahlreicher Nachbarwissenschaften zu pflegen und auf diese Weise die Interdisziplinarität der Geographie am Beispiel eines Gemeinschaftswerkes zu belegen. Alle diese Tatsachen brachten unserm Fach Impulse und Ergebnisse, die ohne das Atlaswerk vielleicht nicht so rasch zum Tragen gekommen wären. Gelobt wurde auch inmer wieder die graphische Harmonie der Karten, die gewählten Farbwerte und Farbstufen, bei denen keine schreienden, aber doch optimal aussagekräftigen Töne Verwendung fanden. 'L'Atlas de $1 a$ Suisse est un plaisir pour les yeux", schrieb
J. Reverdin im Journal de Genève (13.1.1967). So spiegelt sich hier erneut das überragende Wissen und Können von Eduard Imhof.

Es war der Redaktion und der Herausgeberin von Anfang an klar, dass die Fertigstellung nicht das Ende der Arbeiten bedeuten kann, sondern dass der Atlas fortgesetzt werden muss. Wohl bewahren viele Tafeln dauern ihre Aktualität, andere jedoch sind neueren statistischen Erhebun-. gen, wie Volkszählungen, Wirtschaftsstatistiken folgend, erneut zu bearbeiten. Ausserdem sollen einige als richtig anerkannte wïnsche nach Ergänzungen in Form zusätzlicher Tafeln erfüllt werden. Auch bei der Darstellung von kulturgeographischen Belangen sind ohnehin neue Ergebnisse der erdkundlichen Wissenschaft zu erwarten, die kartographischer Darstellung rufen. Wenn neue Tafeln erscheinen, wird vielleicht mancher Atlasbesitzer die alten gegen die neuen austauschen; für den Wissenschafter aber behalten die Erstblätter selbstverständlich ihren dokumentarischen Wert, wird doch der Vergleich des Alten mit dem Neuen mannigfache Entwicklungstendenzen erkennen lassen.

1978 beschloss der Bundesrat, einem Atrag der Redaktionskommission folgend, die Arbeiten in unserm Sinne weiterführen zu lassen. Das Atlasbüro ist im neuen Kartographischen Institut der ETHZ integriert, die personellen Posten und die Finanzierung sind gesichert. Die Redaktionskommission ist in bisheriger proportionaler Zusammensetzung verjüngt, der neue Präsident Prof. Ernst Spiess, Vorsteher des Kartographischen Instituts und Nachfolger Imhofs, war seit 1966 Mitglied der alten Redaktionskommission und bietet Gewähr für sinngemässe Fortsetzung. In seinem Institut stehen überdies neue Apparaturen zur Verfügung, die den Arbeitsaufwand zu beschleunigen erlauben. Die Geographen selber und ihr Verband, wie auch die Kartographen und die vielen zugewandten Wissenschafter, dürfen sich also freuen, dass unser Atlas weiterhin gedeihen wird.

\section{Literatur}

1 Annaheim, A: Geographischer Exkursionsführer der Schweiz. Geographica Helvetica XVI, Bern 1961

2 Annaheim, H. \& Bartsch, D: Gempenplateau und angrenzende Teilregionen. Geographica Helvetica XVIII, Bern 1963

3 Grosjean, G: Exkursionsraum Bern - Längenberg - Schwarzenburg - Sensegraben. Geographica Helvetica XVI, Bern 1961

4 Gutersohn, H: Schweizerischer Landesatlas. Vierteljahrsschrift der Naturforschenden Gesellschaft in Zürich, Zürich 1943

5 Gutersohn, H: Die Geographie im Atlas der Schweiz. Fachblatt Vermessung, Photogrammetrie, Kulturtechnik, l-75 . Zürich 1975

6 Haas, H: Exkursionsführer. Der Schweizer Geograph, 15. Jg., Bern 1938

7 Imhof, E: Ein schweizerischer geographischer Landesatlas. Archiv für das schweizerische Unterrichtswesen; 27, Frauenfeld 1941

8 Imhof, E: Atlas national Suisse. Le Globe, 93, Genève 1944

9 Imhof, E: Der schweizerische Landesatlas. Geographica Helvetica XVII, Bern 1962

10 Imhof, E. u.a.: Der Atlas der Schweiz. Geographica Helvetica XX, Bern 1965

11 Imhof, E: Der Atlas der Schweiz. Internationales Jahrbuch für Kartographie, VI, Gütersloh 1966

12 Imhof, E: Die geomorphologischen Karten im Atlas der Schweiz. Geographica Helvetica XXVII, Bern 1972

13 Meylan, R: La Vallée de Joux. Geographica Helvetica XIX, Bern 1964 
14 Roth-Kim, J: Schweizerische Landesatlanten aus dem 19. und dem frühen 20. Jahrhundert. Geographica Helvetica XXI, Bern 1966

15 Vosseler, P: Atlas de France. Der Schweizer Geograph, 11. Jg., Bern 1934

16 Vosseler, P: Ein schweizerischer Nationalatlas. Der Schweizer Geograph, 18. Jg., Bern 1941

17 Winkler, E: Die kartographischen Darstellungen der schweizerischen Landesausstellung 1939 und die Frage eines geographischen Landesatlasses. Der Schweizer Geograph, 17. Jg., Bern 1940
18 Winkler, E: Landeskunde, Geographie und schweizerischer Landesatlas. Der Schweizer Geograph, 18. Jg., Bern 1941

19 Winkler, E: Regionalbeschreibungen der Schweiz als Grundlage geographischer Landesaufnahme und Landesplanung. Geographica Helvetica VIII, Bern 1953

20 Winkler, E: Ein Raumplanungsatlas der Schweiz. Fachblatt Vermessung, Photogrammetrie, Kulturtechnik, 1-75 , Zürich 1975

21 Wirth, w: Bausteine zu einem geographischen Exkursionsführer des Kantons Zürich. Der Schweizer Geograph, 16. Jg., Bern 1939

\section{Literaturbesprechung}

IMHOF, Eduard: Malerisches Relief des klassischen Bodens der Schweiz von Friedrich Wilhelm Delkeskamp. 17 S., div. Kartenbeilagen (Faksimiledrucke), Verlag Bibliophile Drucke von Josef Stocker AG, Dietikon, 1978.

Zu Beginn der 1930er Jahre, als nicht nur Wissenschaft und Dichtung sondern auch Malerei und Reiseschriftstellerei gewaltig an Bedeutung gewonnen hatten, erschien ein Kartenwerk, das dem zunehmenden Fremdenstorm der damaligen Zeit eine willkommene Hilfe bedeutete, fehlte es doch weitgehend an genauen und bildhaften Karten aller Art .

Ermutigt durch den grossen Erfolg seines kurz zuvor erschienenen "Panorama des Rheins von Mainz bis Köln" wollte der Deutsche Delkeskamp auch hier in die Lücke springen und wenigstens einen wichtigen Teil der Schweiz kartieren. Es handelt sich um den Raum zwischen Pilatus-Glärnisch-Thalwil und Wassen, ein Gebiet von $3.760 \mathrm{~km}^{2}$, aufgeteilt in 9 Karten von $43 \times 28 \mathrm{~cm}$ Grösse, in den Massstäben 1:45'000 W-E und 1:80'000 N-S. Das Werk zeigt den Raum in schräger Vogelschauansicht mit Blick von Norden. Es basiert auf dem "Atlas Suisse", dem besten Kartenwerk der Schweiz der danaligen Zeit, dem eifrigen Verkehr mit besten Kennern des Landes, vor allem mit Heinrich Keller und nicht zuletzt auf der eigenen topographischen Aufnahme des Gebietes. Von über 700 Standorten grossenteils auf Gipfeln der Bergc, hat Delkeskamp während vier Sommern in uner- müdlicher Kleinarbeit den Rohbau für sein malerisches Relief aufgezeichnet. Er gravierte eigen, händig das gesamte Linien- und Konturenbild sowie die topographischen Details seiner Blätter, während Franz Hegi und J.J.Sperli die Aquatintabearbeitung besorgten. Wegen preislicher Schwierigkeiten konnten nur einzelne Blätter mit Aquarellfarben übermalt werden. Eines davon liegt der im Stocker Verlag in hervorragender Präsentation herausgegebenen Faksimile-Ausgabe bei.

Das Werk besticht nicht nur durch seine Naturähnlichkeit der Geländeformen, sondern auch durch seinen Reichtum an Einzelheiten. Häuser, Kirchen, Burgen, Klöster, ehemalige Schlachtfelder, agrarische Flurformen, Strassen und Wege, Obsthaine und Wälder, alles ist minutiös eingezeichnet. Nummern und Buchstaben im Bildinneren und die zugehörigen Erläuterungen an den Blatträndern erleichtern die Orientierung.

Es ist das grosse Verdienst unserers Altmeisters und unermüdlichen Förderers der Kartographie, Eduard Imhof, alles zusammengetragen zu haben, was sich über Delkeskamp auftreiben liess und so der heutigen Generation ein Werk vorzulegen, das auf Schritt und Tritt das ländliche Bild der Innerschweiz in den frühen Jahrzehnten des 19. Jahrhunderts offenbart, Jahre vor dem Bau der Eisenbahn, vor dem eigentlichen Industriezeitalter, dem starken Wachstum det Städte, dem Bau der modernen Verkehrswege und der Verbauung vieler Flüsse.

GH 4/79
Hans Bernhard, Zürich 